\title{
ECLETICA
}

WWW.scielo.br/eq

Volume 30, número 4, 2005

\section{Determinação simultânea de NADH e ácido ascórbico usando voltametria de onda quadrada com eletrodo de carbono vítreo e calibração multivariada}

\author{
R. C. Barthus ${ }^{1}$, L. H. Mazo ${ }^{1 *}$, R. J. Poppi ${ }^{2}$ \\ ${ }^{1}$ Instituto de Química de São Carlos, Universidade de São Paulo, C.P. 780, CEP 13560-970, São Carlos, SP. \\ ${ }^{2}$ Departamento de Química Analítica, Instituto de Química, Universidade Estadual de Campinas, C.P. 6154, CEP \\ 13083-862, Campinas, SP. \\ *Corresponding author
}

\begin{abstract}
Resumo: O método dos mínimos quadrados parciais (PLS) foi aplicado aos dados obtidos por voltametria de onda quadrada para a determinação simultânea de ácido ascórbico (AA) e do co-fator b-nicotinamida adenina dinucleotídeo (NADH) em misturas sintéticas. As curvas voltamétricas foram obtidas em tampão fosfato $0,2 \mathrm{~mol} \mathrm{~L}^{-1}(\mathrm{pH} \mathrm{8,0)}$. Foi possível verificar que o método PLS permite a determinação destes compostos simultaneamente nas condições escolhidas. Os resultados mostraram um erro relativo máximo de $1,7 \%$ para o NADH e 2,1\% para o AA. A metodologia proposta é simples e desenvolvimentos posteriores podem torná-la útil para análises in vivo.
\end{abstract}

Palavras-chave: NADH; ácido ascórbico; mínimos quadrados parciais (PLS); voltametria de onda quadrada.

\section{Introdução}

O cofator b-nicotinamida adenina dinucleotídeo (NADH) é uma importante coenzima, tendo ação catalítica em mais de trezentos processos enzimáticos [1-3]. A detecção do NADH em fluidos biológicos é particularmente importante porque permite a determinação indireta de várias substâncias não-eletroativas presentes in vivo. Em virtude disso, um grande número de pesquisas visando o desenvolvimento de procedimentos analíticos para a determinação de NADH têm sido realizadas. Dentre os vários métodos existe um interesse particular para aqueles envolvendo a oxidação eletroquímica do NADH em meio aquoso [4].

O NADH sofre oxidação para a produção de $\mathrm{NAD}^{+}$com perda de dois elétrons. O mecanismo desta oxidação é complexo, depende de sobrepotenciais de eletrodos elevados e o produto da oxidação pode bloquear a superfície do eletrodo, o que resulta em respostas pouco reprodutivas. Segundo dados da literatura, uma das formas de se melhorar a resposta eletroquímica de compostos como o NADH é pelo uso de catalisadores que acelerem a cinética de transferência de elétrons, ou via catálise heterogênea com o uso de eletrodos modificados e ainda reduzindo o sobrepotencial da reação trabalhando-se em valores mais altos de $\mathrm{pH}$ [4-5]. A aplicação de eletrodos modificados apresenta duas dificuldades importantes na detecção do NADH : a primeira é o bloqueio da superfície do eletrodo por produtos de oxidação que ocorre depois de pouco tempo da polarização do eletrodo e o segundo problema é a tendência em mediar a oxidação eletroquímica do ácido ascórbico (AA) que é o principal interferente presente em sistemas biológicos [4]. Dessa forma, estudos estão sendo realizados para produzir eletrodos modificados capazes de discriminar entre as reações de oxidação de NADH e AA e que sejam suficientemente imunes aos efeitos de adsorção. Alguns eletrodos modificados conseguem resolver o problema da interferência eletroquímica na determinação destes compostos, entretanto, este aumento na seletividade é acompanhado por uma 
grande diminuição na sensibilidade. Em um dos trabalhos citados da literatura [6], um eletrodo de diamante dopado com boro muito sensível é utilizado para a determinação do NADH e ácido ascórbico. O potencial de oxidação do ácido ascórbico e do NADH neste eletrodo difere por $200 \mathrm{mV}$, sendo que mesmo esta diferença de potenciais de pico não permite que haja uma resolução adequada dos picos voltamétricos. Neste caso, a presença de ácido ascórbico é manifestada como um ombro no pico do NADH.

O objetivo deste trabalho foi verificar a possibilidade da aplicação do método multivariado baseado em mínimos quadrados parciais - PLS (do inglês Partial Least Square) para a análise destes compostos em misturas binárias. Este estudo pode ser potencialmente útil mesmo quando superados os problemas comuns de adsorção no eletrodo, existe uma resposta inadequada devido à sobreposição de picos. Usando-se métodos multivariados, como o PLS, este último problema pode ser resolvido.

O PLS $[7,8]$ é o método mais comumente utilizado para calibração multivariada. Neste método, os sinais multivariados, como os valores de corrente medidos em diferentes potenciais do voltamograma (variáveis x) e concentrações (variáveis y) são utilizadas para estabelecer um modelo de regressão linear. Para a construção do modelo de calibração, os dados são dispostos na forma de matrizes: matriz $\mathbf{X ~ e ~} \mathbf{Y}$, as quais contêm as variáveis independentes (x) e dependentes (y), respectivamente. Estes dados podem primeiramente passar, ou não, por pré-tratamentos como o de centrar na média, escalonamento, etc . Após, o processo seguinte consiste em decompor as matrizes originais em "scores" e "loadings". É possível representar uma matriz de dados, sem a perda de informação estatística útil, pela sua matriz de "scores", fazendo-se a redução das variáveis pela análise de componentes principais. Diversos algoritmos podem ser utilizados e o modelo resultante é:

$$
\begin{aligned}
& \mathbf{X}=\mathbf{T} \mathbf{P}^{\mathbf{t}}+\mathbf{E}=\sum \mathbf{t}_{\mathbf{f}} \mathbf{p}_{\mathbf{f}}{ }^{\mathbf{t}}+\mathbf{E} \\
& \mathbf{Y}=\mathbf{U} \mathbf{Q}^{\mathbf{t}}+\mathbf{F}=\sum \mathbf{t}_{\mathbf{f}} \mathbf{q}_{\mathbf{f}}{ }^{\mathfrak{t}}+\mathbf{F}
\end{aligned}
$$

onde os elementos das matrizes de $\mathbf{T}$ e $\mathbf{U}$ são os "scores" de X e Y respectivamente e os elementos $\mathbf{P}$ e $\mathbf{Q}$ são os "loadings". As matrizes $\mathbf{E}$ e $\mathbf{F}$ correspondem aos erros, ou seja, a falta de ajuste do modelo. O sobrescrito t indica matriz transposta. Uma relação entre os dois blocos (variáveis independentes $\mathrm{x}$ e variáveis dependentes y) pode ser realizada correlacionando simultaneamente os "scores" dos blocos X e Y. Para que se encontre uma melhor correlação possível torna-se importante manipular a informação desses dois blocos simultaneamente. No PLS isto é feito por uma leve rotação das componentes principais, com consequente mudança nos valores dos "scores". Devido ao fato de ocorrer esta rotação, o termo componente principal já não é mais utilizado, no lugar deste o termo designado é o de variáveis $\begin{array}{lllllllll} & \mathrm{a} & \mathrm{t} & \mathrm{e} & \mathrm{n} & \mathrm{t} & \mathrm{e} & \mathrm{s}\end{array}$ O número de variáveis latentes utilizados no processo de calibração é obtido por meio de validação cruzada que é uma metodologia baseada na magnitude dos erros de previsão de um dado modelo de calibração. Esta avaliação é feita pela comparação das previsões das concentrações previamente conhecidas $\left(\mathrm{y}_{\mathrm{i}}, \mathrm{i}=\ldots \mathrm{n}\right)$. De uma forma geral, o processo é realizado da seguinte maneira: certas amostras são retiradas do conjunto de treinamento usado para a construção do modelo de calibração. Um novo modelo é construído com as amostras que sobraram. A seguir, as amostras que foram retiradas são utilizadas para a previsão, sendo calculados os resíduos referentes a esta previsão ; erros de previsão $\left(\mathrm{y}_{\mathrm{i}}-\mathrm{y}_{\mathrm{i}}\right)$, onde $\mathrm{y}_{\mathrm{i}}$ é o valor estimado pelo modelo para a concentração. O processo é repetido com outros grupos de amostras, até que todas as amostras tenham sido colocadas fora do modelo de calibração. Calculase, então, a soma dos quadrados dos erros de previsão PRESS.

A primeira versão deste método e adotada

$$
\text { PRESS }=\sum_{i=1}^{n}\left(y_{i}-\hat{y}_{i}\right)
$$

neste trabalho consiste em retirar do conjunto de treinamento uma amostra de cada vez. Este método é chamado validação cruzada completa. O processo 
é repetido com uma, duas e assim por diante, variáveis latentes. Para cada sistema em estudo, o número mais adequado de variáveis latentes, $\mathrm{k}$, será o correspondente ao menor valor de PRESS.

A utilização do PLS tem várias vantagens inerentes ao uso das técnicas multivariadas, como o desenvolvimento de um modelo que é menos sensível a ruídos porque vários valores de corrente são utilizados no modelo de calibração ao invés de um único valor e ainda existe a possibilidade de detecção de amostras anômalas ("outliers") que depois de identificadas podem ser removidas do modelo.

A detecção de "outliers" é tão importante quanto a determinação do número de variáveis latentes utilizados na calibração. Para a detecção de amostras anômalas ("outliers") usam-se duas grandezas complementares: "Leverage" e resíduos de Student.

A "Leverage" é uma medida da influência de uma amostra no modelo de regressão. Um valor de "leverage" pequeno para as amostras indica que esta se encontra próxima do centro dos dados e consequientemente tem pouca influência na construção do modelo. Um valor alto para a "leverage" significa que a amostra está distante da média e possui, dessa forma, grande influência para o desenvolvimento do modelo. Amostras com altas "leverages" normalmente têm alta ou baixa concentração do analito, representam interferentes ou ainda problemas nas medidas instrumentais. Uma análise cuidadosa das amostras com altos valores de "leverages" deve então ser realizada para decidir se esta deve ou não ser eliminada. Com isso, temse que as "leverages" indicam se uma amostra é ou não diferente das demais, mas é necessário outro estudo, como a análise conjunta dos resíduos, para se afirmar se esta amostra corresponde realmente a uma anomalia.

Nos modelos de regressão, como o PLS, a "leverage" $\left(h_{\mathrm{ii}}\right)$ pode ser obtida através da equação 3. que corresponde geometricamente a distância de

$\mathrm{h}_{\mathrm{ii}}=\frac{1}{\mathrm{n}}+\left(\mathbf{x}_{\mathrm{i}}-\overline{\mathbf{x}}\right)^{\mathrm{t}}\left(\mathbf{X}^{\mathrm{t}} \mathbf{X}\right)^{-1}\left(\mathbf{x}_{\mathrm{i}}-\overline{\mathbf{x}}\right)$

um objeto para o centro do modelo e das correlações entre as variáveis. Nesta equação,
$\mathbf{X}_{\mathbf{i}}$ é o voltamograma da i-ésima amostra,$\overline{\mathbf{X}}$ é o voltamograma médio e $\left(\mathbf{X}^{\mathrm{t}} \mathbf{X}\right)$ é a matriz de variânciacovariância. Existe uma regra prática que nos permite distinguir amostras anômalas : amostras com, $\mathrm{h}_{\mathrm{ii}}>\mathrm{h}_{\text {crit }}$ onde $\quad \mathrm{h}_{\text {crit }}=\frac{3 \mathrm{k}}{\mathrm{n}} \quad$ são consideradas suspeitas e devem ser analisadas. Nesta expressão, $\mathrm{n}$ é o número de amostras do conjunto de calibração e K é o número de variáveis latentes.

Os resíduos constituem uma outra forma de se detectar anomalias. Estes correspondem a desvios entre os dados de referência e os estimados pelo modelo. Valores de resíduos altos significam que o modelo não está conseguindo modelar os dados de forma adequada. Para se obter a influência de cada amostra em particular, temos o resíduo de student que para amostra i é dado como:

$$
\text { Res. } y_{i}=\sqrt{\frac{\left(y-y_{i}\right)^{2}}{(n-1)\left(1-h_{i}\right)}}
$$

Resíduo de Student $_{i}=\frac{\left(y-y_{i}\right)}{\operatorname{Res} . y \sqrt{1-h_{i}}}$

onde Res. $y_{i}$ é o resíduo da concentração i corrigido pela leverage.

Supondo que os resíduos são normalmente distribuídos pode-se aplicar o teste t para verificar se a amostra está ou não dentro da distribuição com um nível de confiança de $95 \%$. Como os resíduos de Student são definidos em unidades de desvio padrão do valor médio, os valores além de $\pm 2,5$ são considerados altos. Dessa forma, na análise do gráfico de resíduos Student versus "Leverage", deve-se verificar a influência tanto das "leverages" quanto dos "resíduos". Amostras com altos resíduos e pequenos valores de leverage provavelmente apresentam algum erro no valor da concentração que deve de preferência ser medida novamente ou então esta amostra é simplesmente excluída. Amostras com altos resíduos e altas "leverages" correspondem certamente à amostras anômalas e devem ser retiradas do modelo de calibração. 
Depois de estabelecido o número de variáveis latentes e detectada a presença de amostras anômalas, o modelo pode ser estabelecido e destinado à previsão de concentrações de novas amostras. A concentração de novas amostras pode ser estimada a partir de novos "scores" $\mathbf{T}$ * o dos "loadings" $\mathbf{Q}$ do modelo de calibração, o qual pode ser substituído na equação (1), originando a equação (6):

$$
\mathbf{Y}_{\text {new }}=\mathbf{T}^{*} \mathbf{Q}^{\mathrm{t}}
$$

Neste trabalho, após o modelo ter sido desenvolvido este foi aplicado para a determinação simultânea de amostras sintéticas de NADH e ácido ascórbico.

\section{Material e métodos}

Reagentes e Soluções

Todos os reagentes empregados foram de grau analítico e utilizados sem tratamento de purificação prévia. $\mathrm{O}$ cofator b-nicotinamida adenina dinucleotídeo (NADH) e o ácido ascórbico (VC) foram de procedência da Sigma Chemical Co (St Louis, MO, USA). Dihidrogenofosfato de sódio ou fosfato ácido de sódio (Merck) e ácido fosfórico (Merck) foram usados para preparar soluções tampão $0,2 \mathrm{~mol} \mathrm{~L}^{-1}, \mathrm{pH} 8,0$.

Soluções estoques foram preparadas diariamente e utilizadas por um período máximo de duas horas, pela dissolução de NADH e ácido ascórbico em solução tampão. A concentração final da solução estoque foi de $2,32 \mathrm{~g} \mathrm{~L}^{-1}$ para NADH e 2,04 $\mathrm{g} \mathrm{L}^{-1}$ para o ácido ascórbico.

Água deionisada e purificada em um sistema Milli-Q da Millipore foi utilizada em todos os procedimentos.

\section{Instrumentação}

Todas as medidas voltamétricas de onda quadrada foram realizadas em um analisador polarográfico da EG\&G Princeton Applied Research (PAR) modelo 174 A que foi automatizado pelo uso de uma interface "Lab made" (12bits A/D \& D/A) conectado em um microcomputador IBM/PC onde os dados digitalizados correspondentes às ondas voltamétricas foram registrados e posteriormente submetidos aos tratamentos estatísticos. As curvas de onda-quadrada para as amostras foram registradas na faixa de potencial de $-500 \mathrm{mV}$ a 1300 $\mathrm{mV}$ e a partir destes dados um número apropriado de pontos foi utilizado para compor a matriz para cálculos de PLS.

Foi utilizado um sistema convencional de três eletrodos consistindo de um eletrodo de carbono vítreo como eletrodo de trabalho, um eletrodo de $\mathrm{Ag} / \mathrm{AgCl}_{(\mathrm{s})} \quad\left(\mathrm{KCl}-3,0 \mathrm{~mol} \mathrm{~L}^{-1}\right)$ de referência e um contra-eletrodo de platina posicionados adequadamente na célula eletroquímica. Precedendo todas as medidas voltamétricas, o eletrodo de trabalho foi polido com alumina e lavado com água Milli-Q em abundância. Os parâmetros otimizados utilizados para as análises de voltametria de onda quadrada foram: freqüência $100 \mathrm{~Hz}$, amplitude do pulso $55 \mathrm{mV}$ e incremento do pulso de $2 \mathrm{mV}$.

\section{Software}

O PLS toolbox, versão 2.0 (Eigenvector Technologies, Manson, USA) do software MATLAB $^{\circledR}$, versão 6.0 (The Mathworks, Natik, USA), foi utilizado para o processamento dos dados.

Conjunto de amostras utilizadas na Calibração e na Validação

Para fins de calibração, misturas das amostras sintéticas de ácido ascórbico (AA) e NADH em tampão fosfato $0,2 \mathrm{~mol} \mathrm{~L}^{-1}(\mathrm{pH} 8,0)$ foram utilizadas como padrões, obtendo um total de 25 soluções. A faixa de concentração das amostras variou de $2,32 \mathrm{~g} \mathrm{~L}^{-1}$ a $1,06 \mathrm{~g} \mathrm{~L}^{-1}$ para NADH e de $2,04 \mathrm{~g} \mathrm{~L}^{-1}$ a $0,93 \mathrm{~g} \mathrm{~L}^{-1}$ para o AA. Para fins de validação outras cinco amostras sintéticas com diferentes concentrações de AA e NADH e nas mesmas faixas de concentração, foram utilizadas.

\section{Resultados e discussão}

Os voltamogramas de onda quadrada para o NADH, ácido ascórbico e para a mistura binária NADH e ácido ascórbico em meio a tampão fosfato $0,2 \mathrm{~mol} \mathrm{~L}^{-1}(\mathrm{pH} 8,0)$ são mostrados na figura 1 . O $\mathrm{NADH}$ apresenta um pico de oxidação em potencial 
de $0,62 \mathrm{~V}$ e um outro pequeno pico em potencial de $1,5 \mathrm{~V}$ que não mostra alterações significativas quando do aumento da concentração de NADH. O outro composto, o ácido ascórbico apresenta um pico de oxidação em potencial de $0,38 \mathrm{~V}$ neste sistema. A oxidação eletroquímica do ácido ascórbico gera o ácido dehidroascórbico, com perda de dois elétrons. Pode-se observar que a oxidação eletroquímica do ácido ascórbico, em faixa de potenciais próximas da oxidação do NADH causa interferências nas medidas eletroquímicas. Pela figura 1 pode-se perceber claramente a sobreposição dos picos de oxidação do ácido ascórbico e do primeiro pico de oxidação do NADH. Os produtos de oxidação bloqueiam parcialmente a superfície do eletrodo, tornando necessária a limpeza da superfície, que foi realizada por polimento com alumina antes de cada medida. Utilizando-se este procedimento os voltamogramas apresentaramse reprodutíveis. O tampão fosfato de $\mathrm{pH} 8,0$ foi escolhido porque nestas condições observa-se uma resolução um pouco melhor dos picos de oxidação dos compostos sobre o eletrodo de carbono vítreo sem perda apreciável de sensibilidade da resposta para o NADH.

O modelo de calibração foi baseado na utilização dos dados referente ao primeiro pico de oxidação do NADH e do pico de oxidação do AA. Em análise multivariada é importante que tenhamos somente algum tipo de informação dos analitos presentes e dessa forma, pôde-se fazer uma análise quantitativa desta mistura com bons resultados neste meio.

O método dos mínimos quadrados parciais (PLS) usando amostras sintéticas foi construído com os dados correspondentes aos valores de corrente medidos em diferentes potenciais do voltamograma de onda quadrada (variáveis independentes, x) e os dados de concentração (variáveis dependentes, y). A determinação foi específica para cada composto. Depois de dispostos na forma de matrizes: Matriz $\mathbf{X}$ (correspondentes a dados de variáveis, $\mathrm{x}$ ) e Matriz $\mathbf{Y}$ (correspondentes a dados de variáveis, y), os dados foram submetidos ao pre-tratamento de centrar na média. Este pretratamento corresponde a fazer com que para cada variável seu valor tenha médio zero. Isto é feito, obtendo-se o valor médio para cada variável e subtraindo-o de cada um dos termos da variável correspondente. Isto torna os dados mais bem condicionados para a construção do modelo.

O número de variáveis latentes foi escolhido por validação cruzada, sendo utilizadas três variáveis latentes para a construção do modelo PLS referente ao ácido ascórbico e quatro variáveis latentes foram necessárias para construir o modelo referente ao NADH.

A presença ou não de amostras anômalas foi verificada pela utilização dos gráficos de resíduo Student (SR) versus "Leverage" mostrados nas figuras 2 e 3 para o ácido ascórbico e o NADH respectivamente. A presença de amostras anômalas é caracterizada pela presença simultânea de altos leverage e altos resíduo Student. O gráfico referente ao composto ácido ascórbico mostra que todos as amostras estão dentro da faixa permitida para os valores de resíduo de Student $(2,5)$ e que também não apresenta nenhuma amostra com valor de "leverage" maior que que neste caso corresponde a 0,4 . No gráfico referente ao composto NADH embora as amostras estejam dentro da faixa de erro permitida, uma delas, no entanto apresenta um valor de "leverage" maior que o valor que neste caso é de 0,5. Observa-se que esta amostra, no entanto têm um valor baixo de resíduo e portanto uma análise mais cuidadosa foi realizada. O modelo construído com ou sem esta amostra não apresentou resultados diferentes. Verificou-se que a correspondente amostra contém um valor de

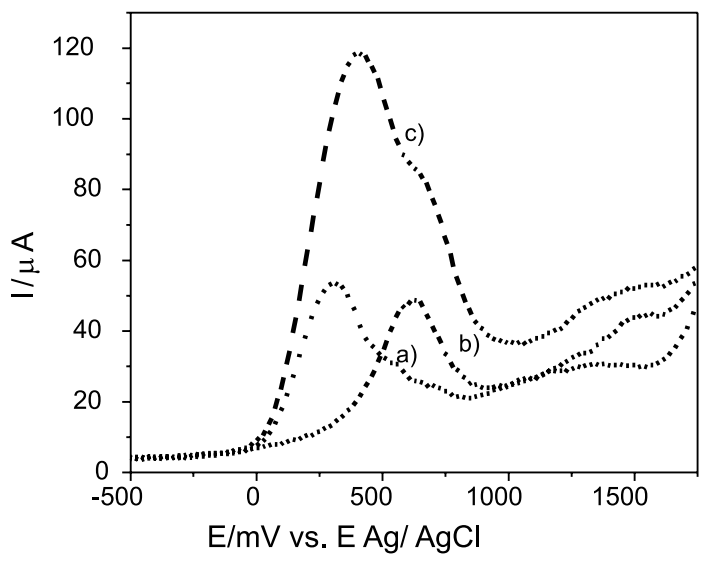

Figura1. Voltamograma de onda quadrada de a) $1,0 \mathrm{~g} \mathrm{~L}^{-1}$ ácido ascórbico, b) $1,20 \mathrm{~g} \mathrm{~L}^{-1} \mathrm{NADH}$ c) mistura de 2,05 $\mathrm{g} \mathrm{L}^{-1} \mathrm{NADH}$ e $1,85 \mathrm{~g} \mathrm{~L}^{-1}$ ácido ascórbico (AA). Condições: $0,2 \mathrm{~mol} \mathrm{~L}^{-1}$ tampão fosfato $\mathrm{pH} 8,0$ frequência $100 \mathrm{~Hz}$, amplitude do pulso $55 \mathrm{mV}$ e incremento do pulso de $2 \mathrm{mV}$. 
concentração somente um pouco diferente das demais e por isso foi conservada no modelo de calibração constituindo-se num dado a mais.

Após estas análises, o modelo construído com o número necessário de variáveis latentes foi utilizado para determinar NADH e ácido ascórbico em outras amostras sintéticas.

Os resultados obtidos com o modelo PLS e os valores para erro relativo e RMSEP (erros de previsão), calculados segundo a equação 7 , são mostrados nas tabelas 1 e 2 para NADH e ácido

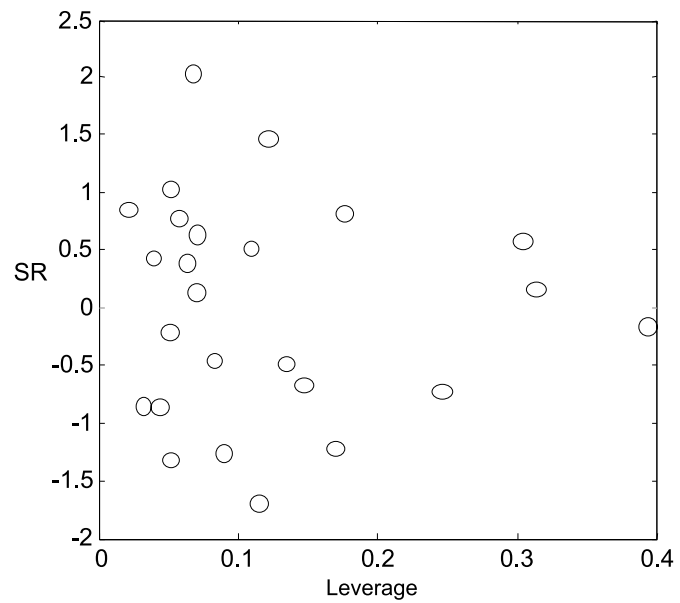

Figura 2. Gráfico de resíduo Student (SR) vs. "Leverage" para o ácido ascórbico. ascórbico respectivamente.

$$
\text { RMSEP }=\sqrt{\frac{\sum\left(\mathrm{y}_{\text {real }}-\mathrm{y}_{\text {prev }}\right)^{2}}{\mathrm{n}}}
$$

onde são os valores reais das concentrações e são os valores estimados pelo modelo e n é o número de amostras.

Pelos resultados apresentados verifica-se que existe boa concordância entre os valores obtidos pelo modelo de calibração e os valores

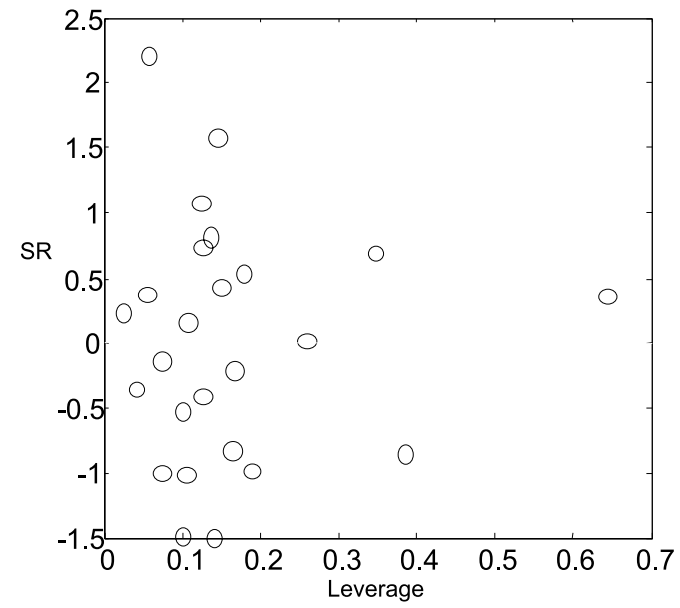

Figura 3. Gráfico de resíduo Student (SR) vs " Leverage" para o NADH.

\section{amostras Valor Real $\left(\mathrm{g} \mathrm{L}^{-1}\right) \quad$ PLS $\left(\mathrm{g} \mathrm{L}^{-1}\right) \quad$ Erro (\%)}

\begin{tabular}{cccc}
\hline 1 & 1,75 & 1,72 & $-1,7$ \\
2 & 1,55 & 1,54 & $-0,6$ \\
3 & 1,33 & 1,35 & 1,5 \\
4 & 1,16 & 1,16 & 0 \\
5 & 1,24 & 1,22 & $-1,6$ \\
RMSEP* & & & $* 1,3$ \\
\hline
\end{tabular}

Tabela1. Resultados obtidos com o modelo PLS para o composto NADH. 


\begin{tabular}{rrrr}
\hline Amostra & Valor Real $\left(\mathrm{g} \mathrm{L}^{-1}\right)$ & PLS $\left(\mathrm{g} \mathrm{L}^{-1}\right)$ & Erro \%) \\
\hline 1 & 1,52 & 1,53 & 0,7 \\
2 & 1,40 & 1,43 & 2,1 \\
3 & 1,17 & 1,16 & $-0,9$ \\
4 & 1,03 & 1,01 & $-1,9$ \\
5 & 0,97 & 0,95 & $-2,1$ \\
RMSEP & & & $* 1,7$ \\
\hline
\end{tabular}

Tabela2. Resultados obtidos com o modelo PLS para o composto ácido ascórbico.

reais das amostras sintéticas, verificando-se que o método pode ser utilizado para a determinação simultânea destes compostos em solução.

\section{Conclusões}

Este trabalho demonstrou que o emprego da técnica voltamétrica de onda quadrada em conjunto com o método quimiométrico PLS produziu bons resultados para a determinação simultânea de ácido ascórbico e NADH em misturas sintéticas. A partir destes primeiros experimentos, pode-se em estudos posteriores empregando microeletrodos ou ultramicroeletrodos verificar a possibilidade do monitoramento de NADH in vivo, o que é de grande importância em muitas investigações fisiológicas.

\section{Agradecimentos}

R.C.Barthus.

À CAPES pela bolsa de doutorado de

Recebido em: 18/08/2005

Aceito em: 27/10/2005

R. C. Barthus, L. H. Mazo, R. J. Poppi. Square wave voltammetric simultaneous determination of NADH and Ascorbic acid using glassy carbon electrode and multivariate calibration.

\begin{abstract}
In this work, the partial least squares (PLS) method was applied to data obtained by square wave voltammetry for simultaneous determination of ascorbic acid and the b-nicotinamide adenine dinucleotide co-factor (NADH) in synthetic mixtures. The voltammetric curves were obtained in $0.2 \mathrm{~mol} \mathrm{~L}^{-}$ ${ }^{1}$ phosphate buffer ( $\mathrm{pH} 8.0$ ). From the results it is possible to verify that the PLS method was able to determine these compounds simultaneously in the choose conditions. The results achieved for these determination provided a maximum value for the relative error of $1.7 \%$ for $\mathrm{NADH}$ and $2.1 \%$ for ascorbic acid. The proposed methodology is simple and further studies can make it useful for in vivo analysis.

Keywords: NADH; ascorbic acid; square wave voltammetry; partial least square (PLS).

\section{Referências}

[1] A. S.N. Murthy, J. Sharma, Talanta 45 (1998) 951.

[2] K.Tanaka, S. Ikeda, N. Oyama, K. Tokuda, Tohsaka, Analytical Sciences 9 (1993) 783.

[3] E.J .Eisenberg, K.C. Cundy , Anal.Chem. 63 (1991) 845.

[4] G. Milczarek, A. Ciszewski, Electroanalysis 13 (2001) 164 .

[5] W.B. Nowall, W.G. Kuhr, Anal. Chem. 67(1995) 3583.
\end{abstract}


[6] T.N.Rao, I.Yagi,T.Miwa, D.A.Tryk, A. Fujishima, Anal. Chem. 71 (1999) 2506.

[7] H. Martens, T. Naes , Multivariate Calibration, John Wiley \& Sons, New York 1989.
[8] B.M. Wise, N.B. Gallagher, PLS-Toolbox 2.1 for use with Matlab, EigenVector Research Inc, W.A Manson,1998. 